УДК 51-7

\title{
РАЗРАБОТКА СИСТЕМЫ УПРАВЛЕНИЯ КОНТЕНТОМ ДЛЯ СОЗДАНИЯ ИНТЕРНЕТ-МАГАЗИНА
}

\author{
Джамбаев Адиль Кусаинович \\ студент \\ Курмангазиева Ляйля Таскалиевна \\ к.т.н., асс. профессор \\ Атырауский университет имени Х. Досмухамедова
}

\begin{abstract}
Аннотация В статье был проведен анализ организации и управления построения систем управления контентом для создания интернет-магазина, были выявлены основные функции, элементы и методы системы управления контентом, а также исследованы процессы планирования, прогнозирования и организации продажами. Таким образом, на основе полученных данных была разработанная система для поддержки торговых на предприятии с внедрённой системой управления контентом, которая повышает эффективность и упрощает работу администратора (продавца), а также клиентов, поскольку позволяет быстро и просто совершать различные покупки, осуществлять поиск необходимых продуктов, а также для удобства пользователя просматривать необходимые продукты по имеющимся категориям.
\end{abstract}

Ключевые слова: интернет-магазин, контент, CMS, CRM, онлайнпродажи, SaaS.

\section{DEVELOPMENT OF A CONTENT MANAGEMENT SYSTEM FOR CREATING AN ONLINE STORE}

\section{Dzhambayev Adil Kusainovich Scientific adviser: Kurmangaziyeva Lyailya Taskalievna}

\begin{abstract}
The article analyzed the organization and management of building content management systems for creating an online store, identified the main functions, elements and methods of a content management system, and investigated the processes of planning, forecasting and sales organization. Thus, based on the data obtained, a system was developed to support trading at the enterprise with an implemented content management system, which increases efficiency and simplifies
\end{abstract}


the work of the administrator (seller), as well as customers, since it allows you to quickly and easily make various purchases, search for the necessary products, and also for the convenience of the user to view the necessary products by available categories.

Key words: online store, content, CMS, CRM, online sales, SaaS.

\section{Постановка задачи на проектирование}

Проанализировав работу множества отделов продаж и онлайнмагазинов, можно сделать вывод о том, что работа большинства из них не автоматизирована и направлена на предоставление удобной работы для одного из лиц: продавца или же покупателя.

Основной задачей проекта является изготовление системы управления контентом для создания интернет-магазином, которая будет проста и удобна в использовании для пользователя, позволяющая быстро и эффективно осуществлять изменение контента администратором, а также предоставлять возможность обычным пользователям осуществлять покупки онлайн с возможностью выбора оптимальных цен и характеристик товара, к тому же продавец будет иметь возможность контроля и учета всех продуктов, заказов, и автоматического расчета оптимальных цен и издержек, которые будут приемлемы, как для самого продавца, так и для покупателя. Данная задача может быть решена посредством совмещения концепций интернет-магазина и CRM-системы.

Система управления взаимоотношениями с клиентами (CRM-система) прикладное программное обеспечение для организаций, предназначенное для автоматизации стратегий взаимодействия с заказчиками (клиентами), в частности для повышения уровня продаж, оптимизации маркетинга и улучшения обслуживания клиентов путём сохранения информации о клиентах и истории взаимоотношений с ними, установления и улучшения бизнеспроцессов и последующего анализа результатов [1].

CRM - модель взаимодействия, главной идеей которой является центром всей философии бизнеса является клиент, а главными направлениями деятельности компании являются меры по обеспечению эффективного маркетинга, обслуживания клиентов и продаж.

Это решение поможет:

- получить общую для компании стандартизированную базу контактов (клиентов, контрагентов); 
- получить готовую и протестированную систему управления контентом;

- эффективно осуществлять контроль качества работы отдела продаж в любой момент времени;

- получить статистику и аналитику эффективности работы с потенциальными клиентами (входящими звонками, запросами);

- планировать повышение качества работы и разрабатывать стратегию развития бизнеса.

Таким образом, основными задачами приложения являются:

- гибкая система управления контентом;

- эффективная фильтрация товаров по характеристикам, необходимым для конкретного клиента;

- расчет оптимальной цены товара, базируясь как на повышение прибыли для продавца, так и на спрос среди клиентов;

- внедрение гибкой системы скидок для покупателей;

- предоставление автоматической системы расчета постоянных и переменных издержек, цены и прибыли на каждый вид товара для продавца.

Данное приложение будет разработано с использованием Salesforce платформы. Salesforce - это CRM платформа для успешной работы с клиентами, предназначенная для продажи, обслуживания, маркетинга, анализа и взаимодействия с клиентами, предоставляемая заказчикам исключительно по модели SaaS [2].

$\mathrm{SaaS}$ - это одна из форм облачных вычислений, модель обслуживания, при которой подписчикам предоставляется готовое прикладное программное обеспечение, полностью обслуживаемое провайдером.

Поэтому основным преимуществом разработки на Salesforce как для клиента, так и для разработчика является отсутствие необходимости установки ПО на рабочие места - доступ к ПО осуществляется через обычный Web-браузер, для клиентов это также радикальное сокращение затрат на развёртывание системы в организации.

Исходя из вышесказанного, единственным минимальным требованием для запуска системы является присутствие Web-браузера на компьютере и наличие интернет соединения.

\section{Информационная модель системы и ее описание}

Для хранения объектов в данном проекте использовалась база данных 
платформы Force.com. База данных Salesforce построена на основе объектнореляционной системы управления базами данных компании Oracle.

Объектно-ориентированные базы данных - базы данных, в которых информация представлена в виде объектов, как в объектно-ориентированных языках программирования. Объектно-ориентированные базы данных обычно рекомендованы для тех случаев, когда требуется высокопроизводительная обработка данных, имеющих сложную структуру. Основные трудности объектно-ориентированного моделирования данных проистекают из того, что такого развитого математического аппарата, на который могла бы опираться общая объектно-ориентированная модель данных, не существует. В большей степени поэтому до сих пор нет базовой объектно-ориентированной модели.

Объектно-ориентированный подход базируется на концепциях:

- объекта и идентификатора объекта;

- атрибутов и методов;

- классов;

- иерархии и наследования классов.

Любая сущность реального мира в объектно-ориентированных языках и системах моделируется в виде объекта. Любой объект при своем создании получает генерируемый системой уникальный идентификатор, который связан с объектом во все время его существования и не меняется при изменении состояния объекта.

Каждый объект имеет состояние и поведение. Состояние объекта набор значений его атрибутов. Поведение объекта - набор методов (программный код), оперирующих над состоянием объекта. Значение атрибута объекта - это тоже некоторый объект или множество объектов. Состояние и поведение объекта инкапсулированы в объекте; взаимодействие между объектами производится на основе передачи сообщений и выполнении соответствующих методов[3].

Множество объектов с одним и тем же набором атрибутов и методов образует класс объектов. Объект должен принадлежать только одному классу (если не учитывать возможности наследования, см. следующий абзац). Допускается наличие примитивных предопределенных классов, объектыэкземляры которых не имеют атрибутов: целые, строки и т.д. Класс, объекты которого могут служить значениями атрибута объектов другого класса, называется доменом этого атрибута.

Допускается порождение нового класса на основе уже существующего 255 
класса - наследование. В этом случае новый класс, называемый подклассом существующего класса (суперкласса) наследует все атрибуты и методы суперкласса. В подклассе, кроме того, могут быть определены дополнительные атрибуты и методы. Различаются случаи простого и множественного наследования. В первом случае подкласс может определяться только на основе одного суперкласса, во втором случае суперклассов может быть несколько. Если в языке или системе поддерживается единичное наследование классов, набор классов образует древовидную иерархию. При поддержании множественного наследования классы связаны в ориентированный граф с корнем, называемый решеткой классов. Объект подкласса считается принадлежащим любому суперклассу этого класса.

Одной из более поздних идей объектно-ориентированного подхода является идея возможного переопределения атрибутов и методов суперкласса в подклассе (перегрузки методов). Эта возможность увеличивает гибкость, но порождает дополнительную проблему: при компиляции объектноориентированной программы могут быть неизвестны структура и программный код методов объекта, хотя его класс (в общем случае суперкласс) известен. Для разрешения этой проблемы применяется так называемый метод позднего связывания, означающий, по сути дела, интерпретационный режим выполнения программы с распознаванием деталей реализации объекта во время выполнения посылки сообщения к нему. Введение некоторых ограничений на способ определения подклассов позволяет добиться эффективной реализации без потребностей в интерпретации.

Объектно-ориентированные системы управления базами данных (ООСУБД) позволяет работать с объектами баз данных так же, как с объектами в программировании в ООЯП. ООСУБД расширяет языки программирования, прозрачно вводя долговременные данные, управление параллелизмом, восстановление данных, ассоциированные запросы и другие возможности. Некоторые объектно-ориентированные базы данных разработаны для плотного взаимодействия с такими объектноориентированными языками программирования как Python, Java, C\#, Visual Basic .NET, C++, Objective-C и Smalltalk; другие имеют свои собственные языки программирования. ООСУБД используют точно такую же модель, что и объектно-ориентированные языки программирования. 


\section{Спецификация вариантов использования системы}

Наилучшим средством для описания спецификации системы являются диаграммы вариантов использования. Варианты использования предназначены в первую очередь для определения функциональных требований к системе и управляют всем процессом разработки. Все основные виды деятельности, такие как анализ, проектирование, тестирование выполняются на основе вариантов использования[4].

У обоих участников присутствуют идентичные варианты использования: «Поиск товара по категориям» и «Просмотреть детальную информацию о товаре», поскольку каждый из участников имеет возможность осуществления поиска различных видов товара, а также просмотреть полную информации о товаре. Пользователь может просматривать информацию только о своих заказах, администратору же отображаются все имеющиеся в системе заказы.

Основная задача администратора - управление системой, а точнее контроль и своевременное обновление информации о товарах, а также добавление новых, также в задачи администратора входит настройка системы контроля контента, подбор более технически пригодного для пользования отображения страницы. Данные задачи представлены вариантами использования «Добавить продукт», «Изменить продукт» и «Удалить продукт». Также у администратора присутствует такой вариант использования, как «Назначить скидку клиентам».

Назначение скидок подразумевает под собой скидки, которые готова дать компания на определённый продукт. В систему внедрена система скидок в зависимости от общей стоимости предыдущих заказов для клиентов. Администратор может вернуть часть денег пользователю, если пользователь купил продукт по завышенной цене.

В системе присутствует возможность автоматического расчета стоимости продукт, что может осуществить администратор в процессе внедрения нового товара или редактирования старого. Клиент осуществляет покупку товаров в системе интернет-магазина, поэтому для него доступен вариант использования «Заказать товар», который включает в себя просмотр условий заказа и выбор способа доставки товара. Также пользователь имеет возможность сразу оплатить заказ по карточке.

Также пользователю предоставлена возможность просмотра фотографий различных товаров для визуального анализа товаров и выбора наиболее 
подходящего [5].

\section{Описание обобщенного алгоритма}

В начале работы клиенту предлагается выбрать необходимые товары, которые он может отсортировать по определённым критериям. В том случае если клиент выберет большое количество товара, которое превышает имеющееся количество товаров в наличии, клиенту будет показано сообщение о невозможности добавления такого количества товаров в корзину и предложение выбрать другие характеристики для заказа.

Далее клиентом осуществляется выбор способа доставки заказа, имеется два варианта: самовывоз товаров со склада или же доставка. Поскольку доставка является оплачиваемой, многим клиентам предпочтительнее самим забрать необходимые товары со склада, если есть такая возможность.

После чего осуществляется добавление заказа клиентом. Происходят различные проверки на валидность заказа в системе, такие как, например, превышает ли сумма заказа две тысячи долларов, если заказ превышает две тысячи долларов, то он зачисляется как заказ с большой стоимостью. В данном случае администратору приходит сообщение на почтовый ящик с информацией о данном заказе, а для клиента сообщение, что заказ находится в процессе ожидания до тех пор, пока администратор не одобрит его. Также осуществляется проверка, есть ли у клиента скидка на заказ, в этом случае сумма к оплате пересчитывается с учетом скидки, и для клиента выводится сообщение о новой стоимости заказа [6].

Далее в системе производится пересчет коэффициентов и характеристик данного товара на основе изменившихся данных, такие как затраты на хранение, переменные затраты и тому подобное. После завершения всех действий: обновления расчетов и характеристик товара - проверяется, присутствует ли у клиента скидка, если нет, для клиента добавляется минимальная первоначальная скидка, в обратном же случае - скидка для клиента увеличивается на следующий заказ в зависимости от суммы всех предыдущих заказов [7].

После чего заказ является успешно добавленным, и для клиента выводится сообщение об успешном осуществлении добавления заказа в систему. После клиент должен оплатить заказ при помощи карточки с которой в последствии будут списаны деньги, если по какойто причини товар не был предоставлен, администратор может возместить потраченные средства. 


\section{Руководство пользователя}

Система может использоваться менеджером и обычным покупателем. Продемонстрируем все возможности системы на примере двух лиц. Для клиента при входе в системе открывается главное окно со списком всех имеющихся продуктов. Страница предоставляет возможность расширения и сужения категорий для визуально лучшего представления для клиента, а также выбора необходимого числа продуктов для заказа, что продемонстрировано на рисунке 1 и рисунок 2 .

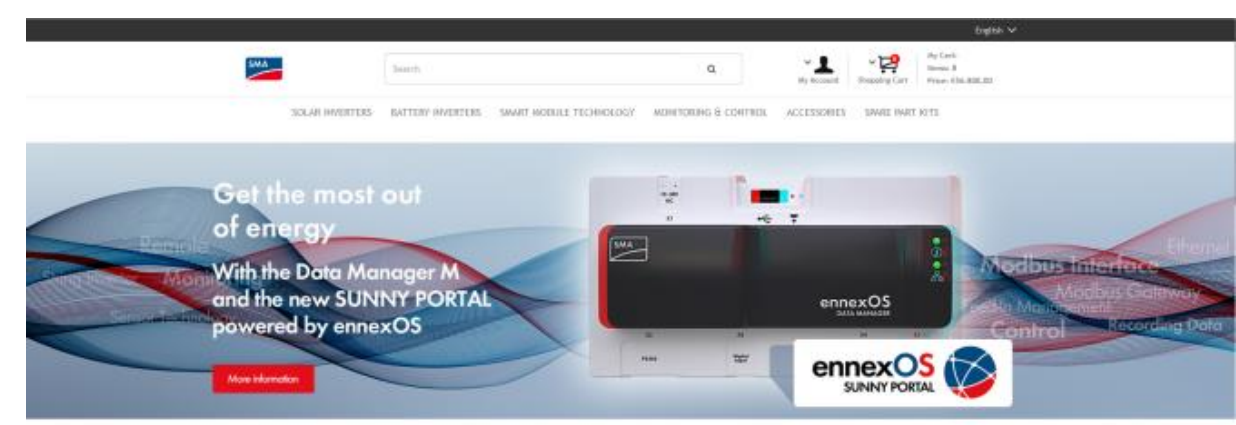

Topseller

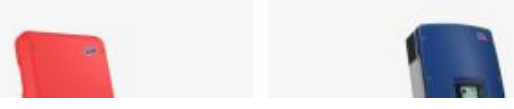

Рис. 1. Страница каталога продуктов

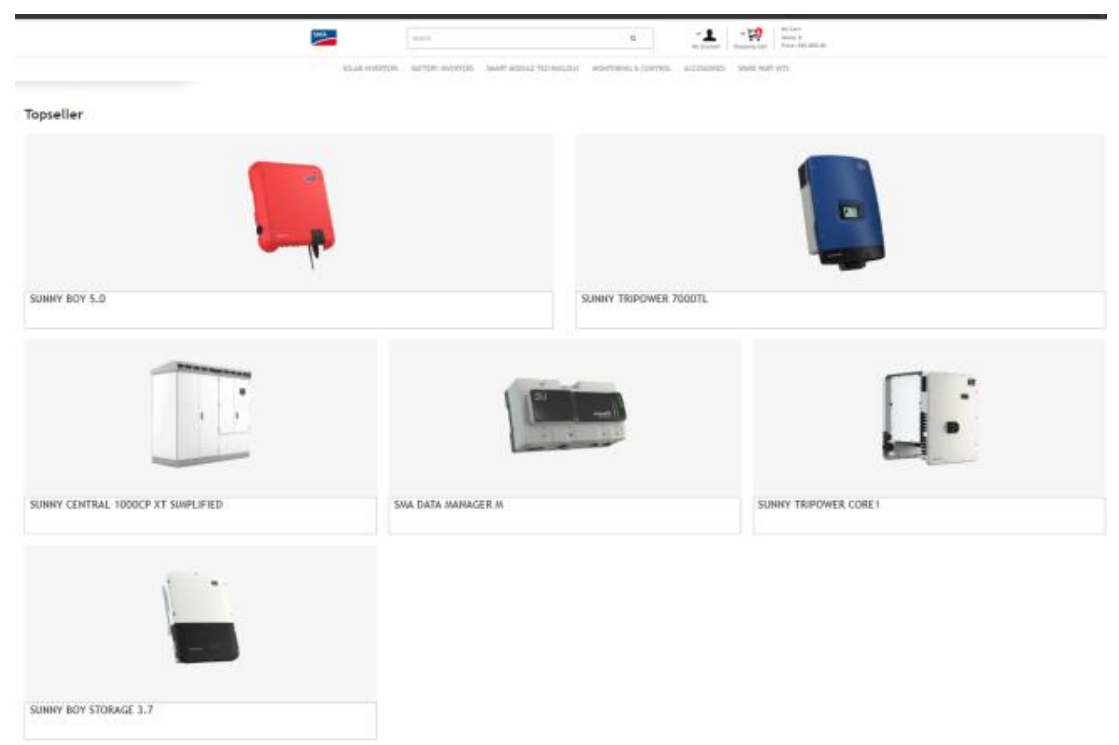

Рис. 2. Страница отображения продуктов

Вверху страницы предоставлены каталоги продуктов по нажатию на который нам открывается отсортированные продукты по определённому 259 
критерию. Также представляется возможность сгруппировать продукты по определённым критериям в правой колонке страницы рисунок 3

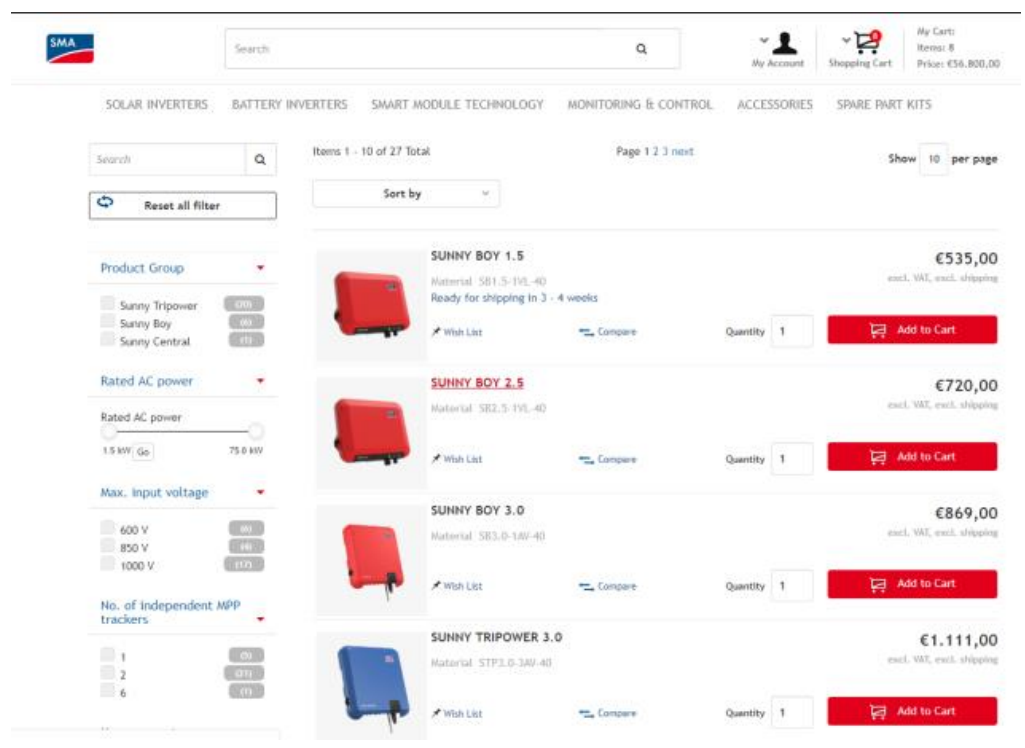

\section{Рис. 3. Страница каталога продуктов Solar Inverters}

Если клиент определился с продуктом, он может добавить нужное количество этого продукта себе в корзину рисунок 4.

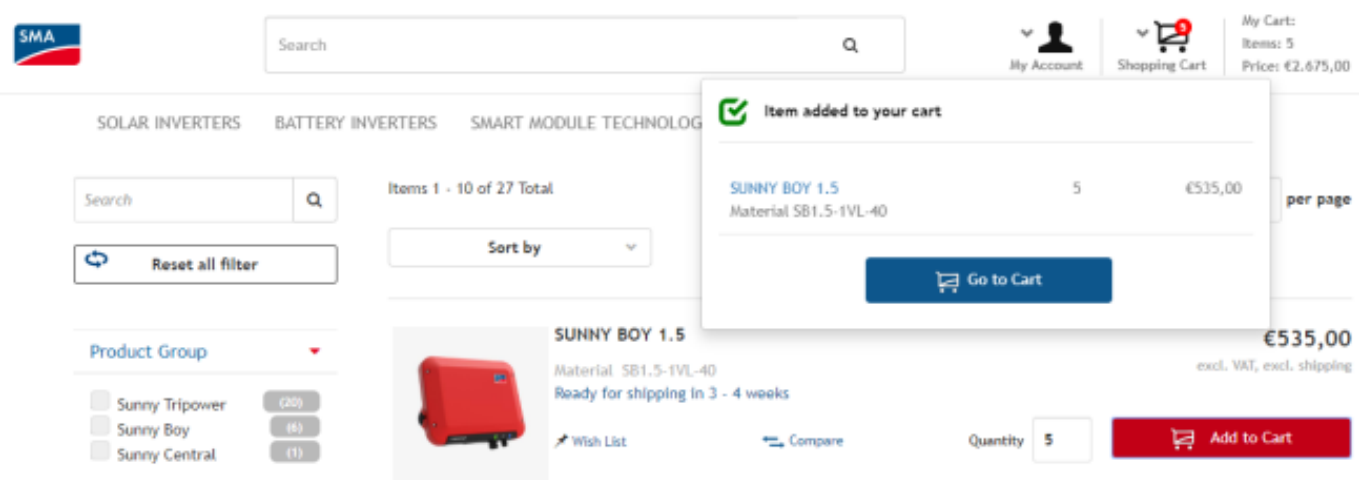

Рис. 4. Добавление продукта в корзину

После перехода в «Shopping cart» клиент может увидеть свои добавления. Он может как удалить из корзины, так и оформить покупку рисунок 5 . 


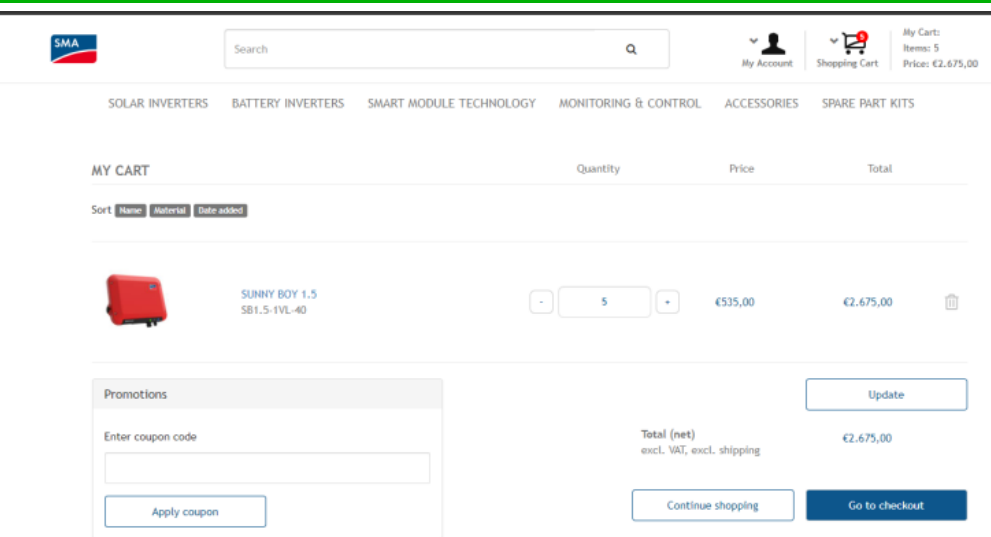

\section{Рис. 5. Страница отображения Shopping Cart}

При нажатии на имя продукта, пользователь может увидеть подробное описание продукта, его плюсы, а также цену. Со страницы подробного описания у пользователя есть возможность добавления этого продукта в корзину рисунок 6 .

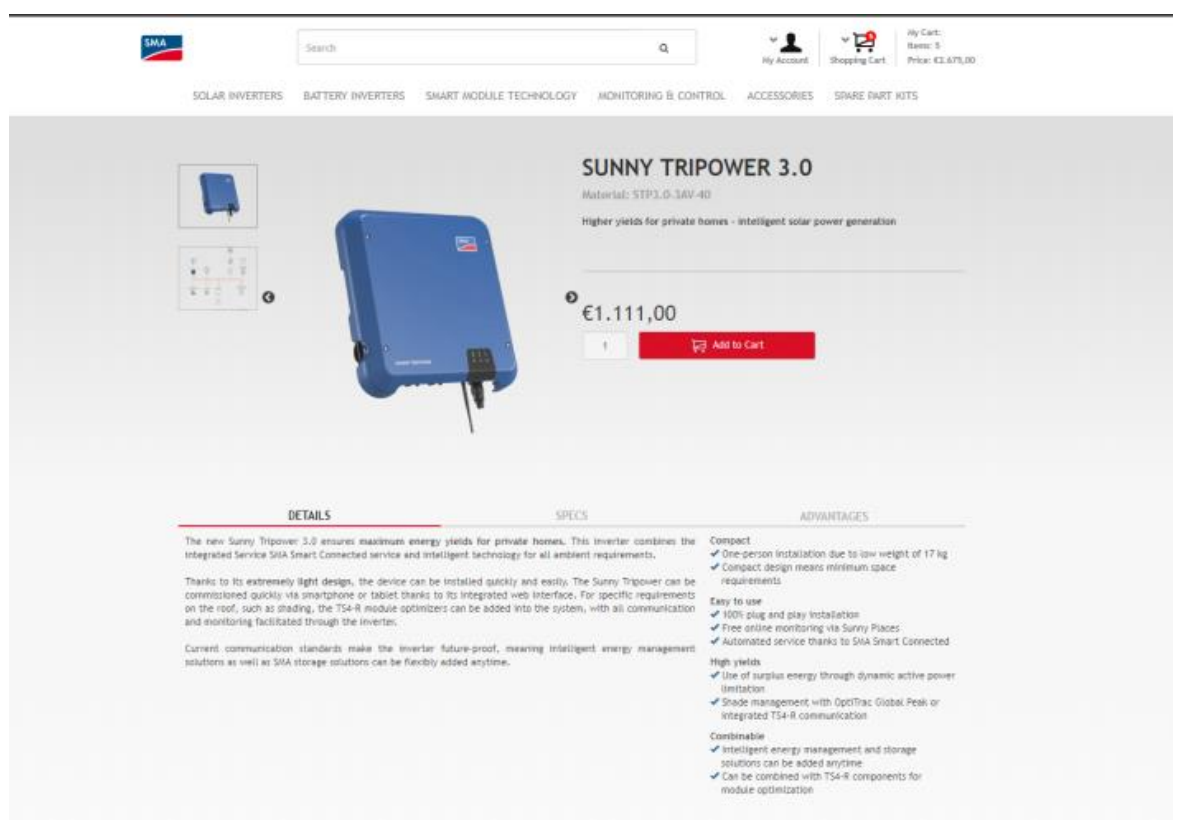

\section{Рис.6. Страница детального описания продукта}

Внизу страницы предоставлены вкладки при нажатии на которые меняется отображение самой страницы. При нажатии на вкладку «Specs» пользователю отображаются подробные характеристики данного продукта рисунок 7. 


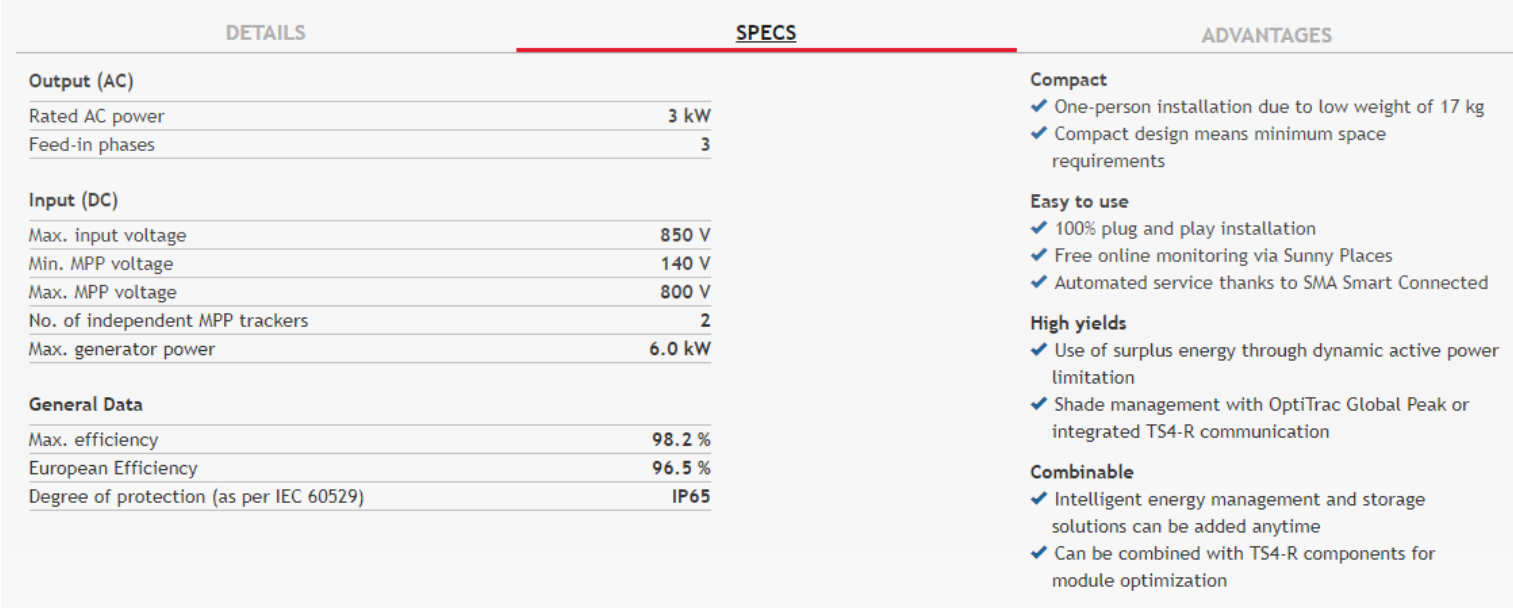

\section{Рис.7. Детальное описание продукта для клиента}

Также ниже отображаются продукты, которые пригодятся при установке выбранного пользователем продукта рисунок 8 .

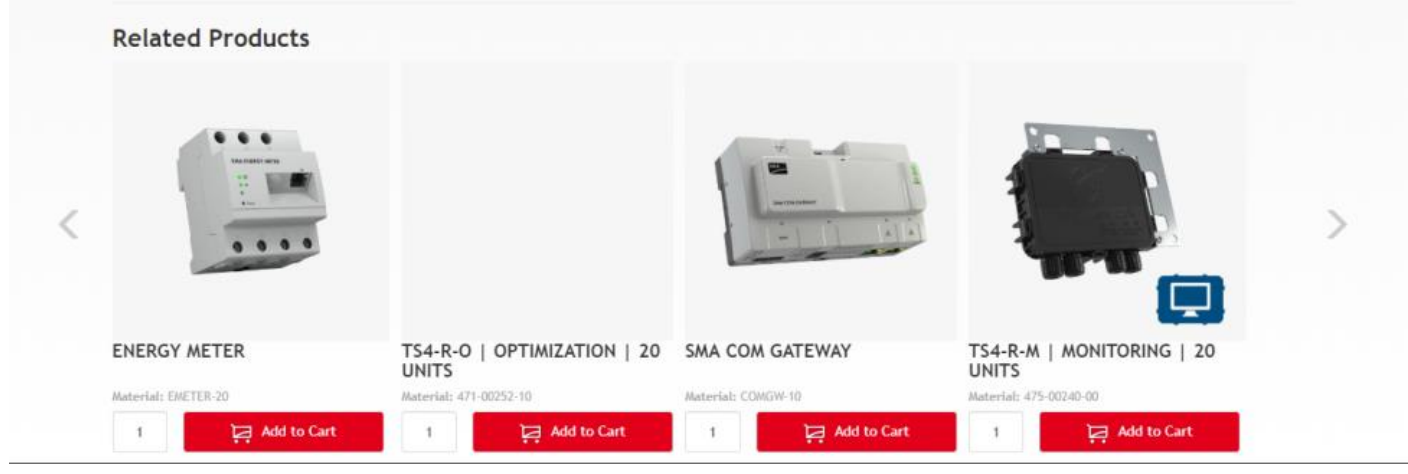

Рис. 8. Лист зависимых продуктов

Вверху страницы предусмотрен выбор языка страницы рисунок 9.

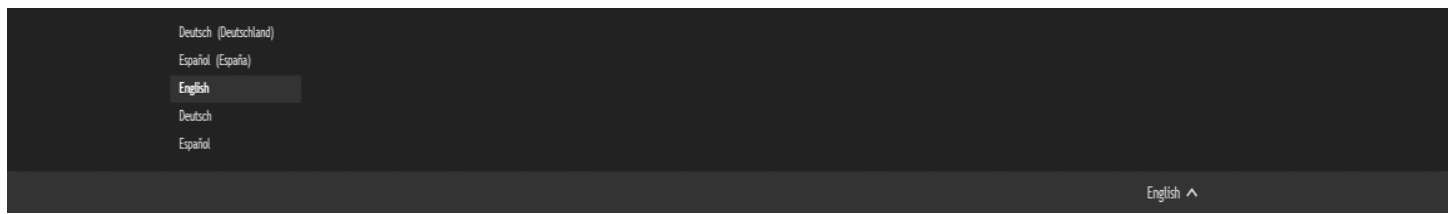

Рис. 9. Выбор языка отображения страницы

После выбора языка отображения, страница поменяет свой язык, что можно увидеть на рисунке 10. 


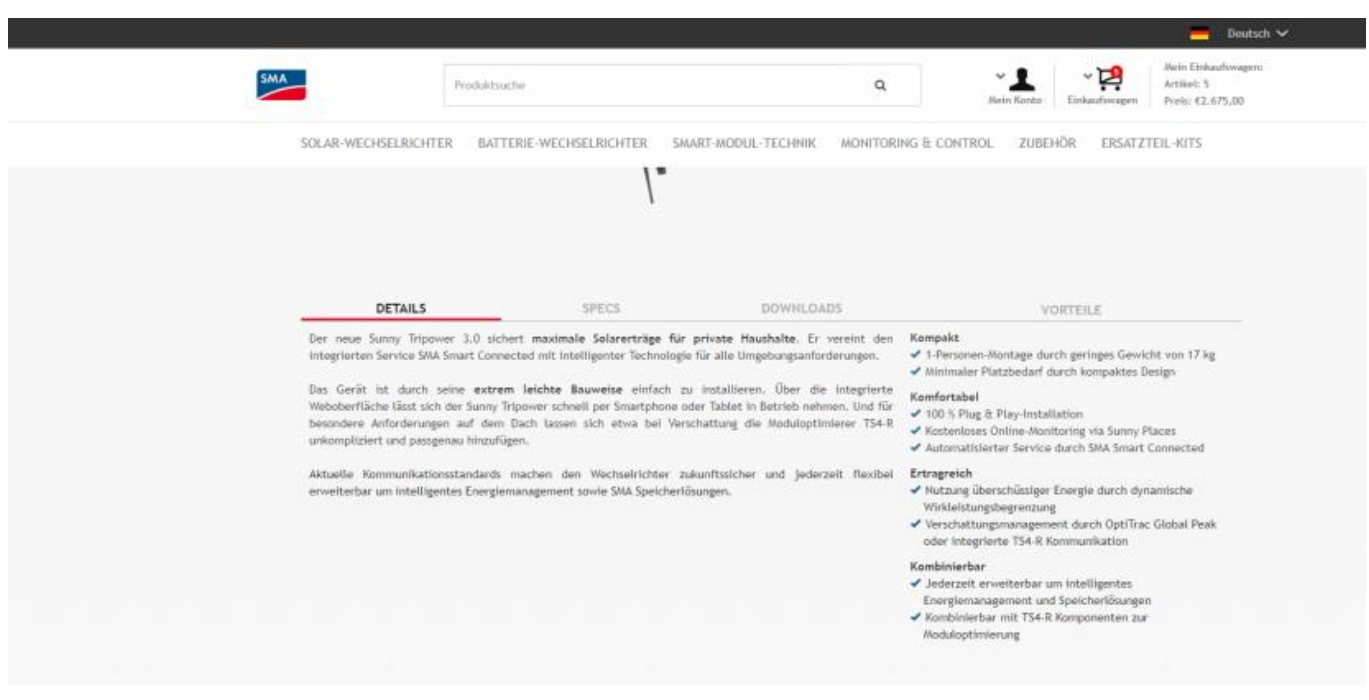

Рис. 10. Изменение языка страницы

После того как пользователь определится с выбором товара и будет готов его купить, ему нужно зайти в корзину и нажать на кнопку «Go Tо Check», после этого его переведёт на страницу оплаты, которая продемонстрирована на рисунке 10.

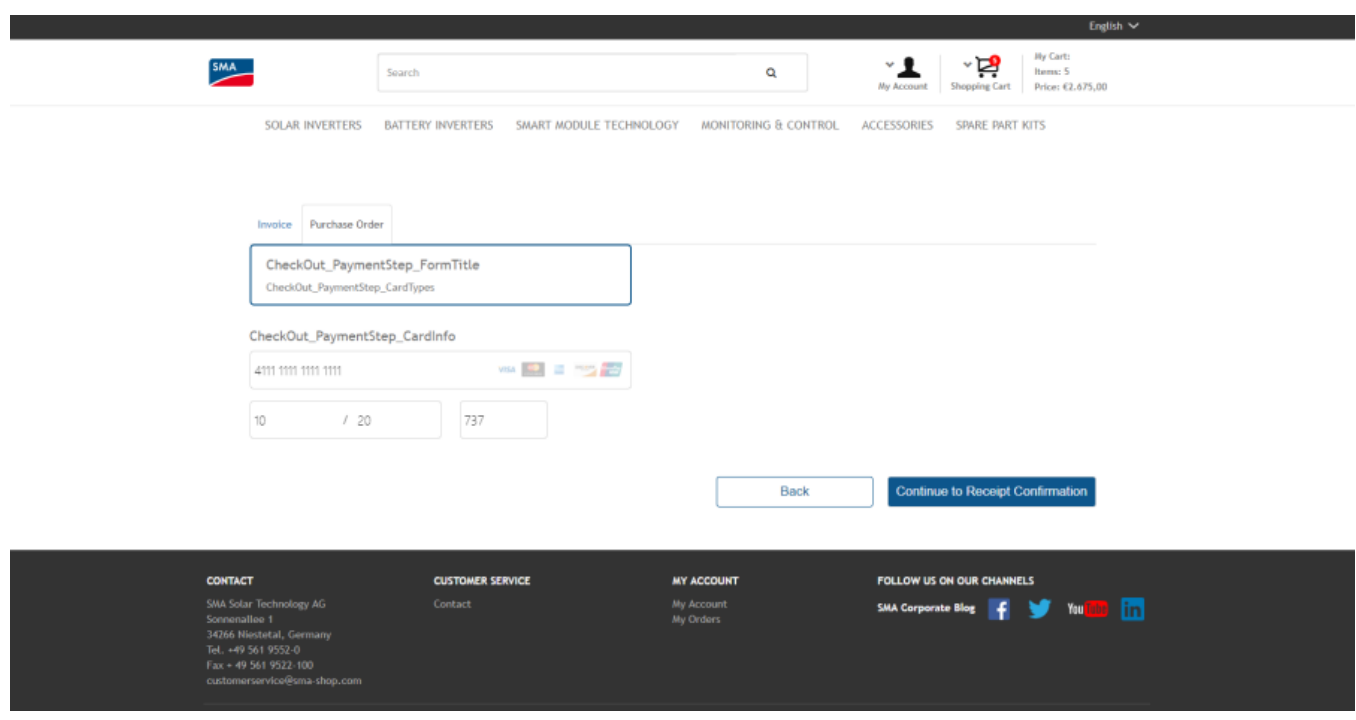

Рис. 11. Оплата продукта

После нажатия на кнопку «Continue to Receipt Confirmation» пользователя переводит на страницу с чеком о покупке выбранного продукта рисунок 11. 


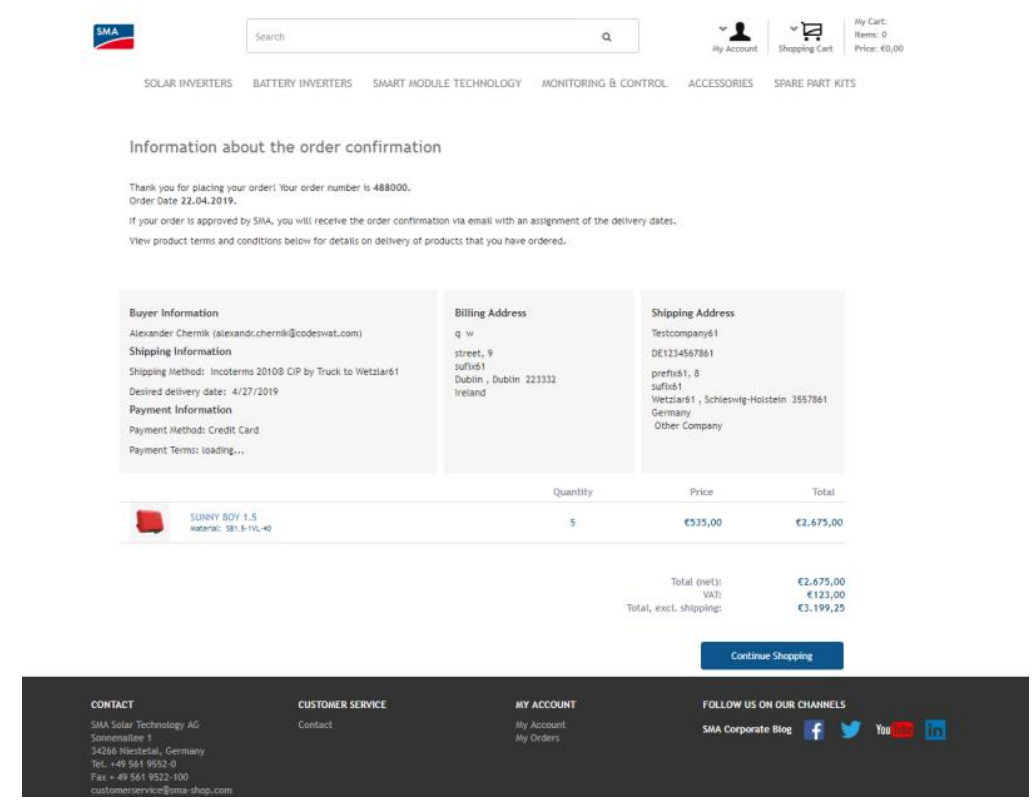

\section{Рис. 12. Страница отображения чека покупки}

Администратор может изменять информацию о продукте, его цену и т.д. это показано на рисунке 12.

Ниже администратор может увидеть графики о покупках данного продукта рисунок 13.

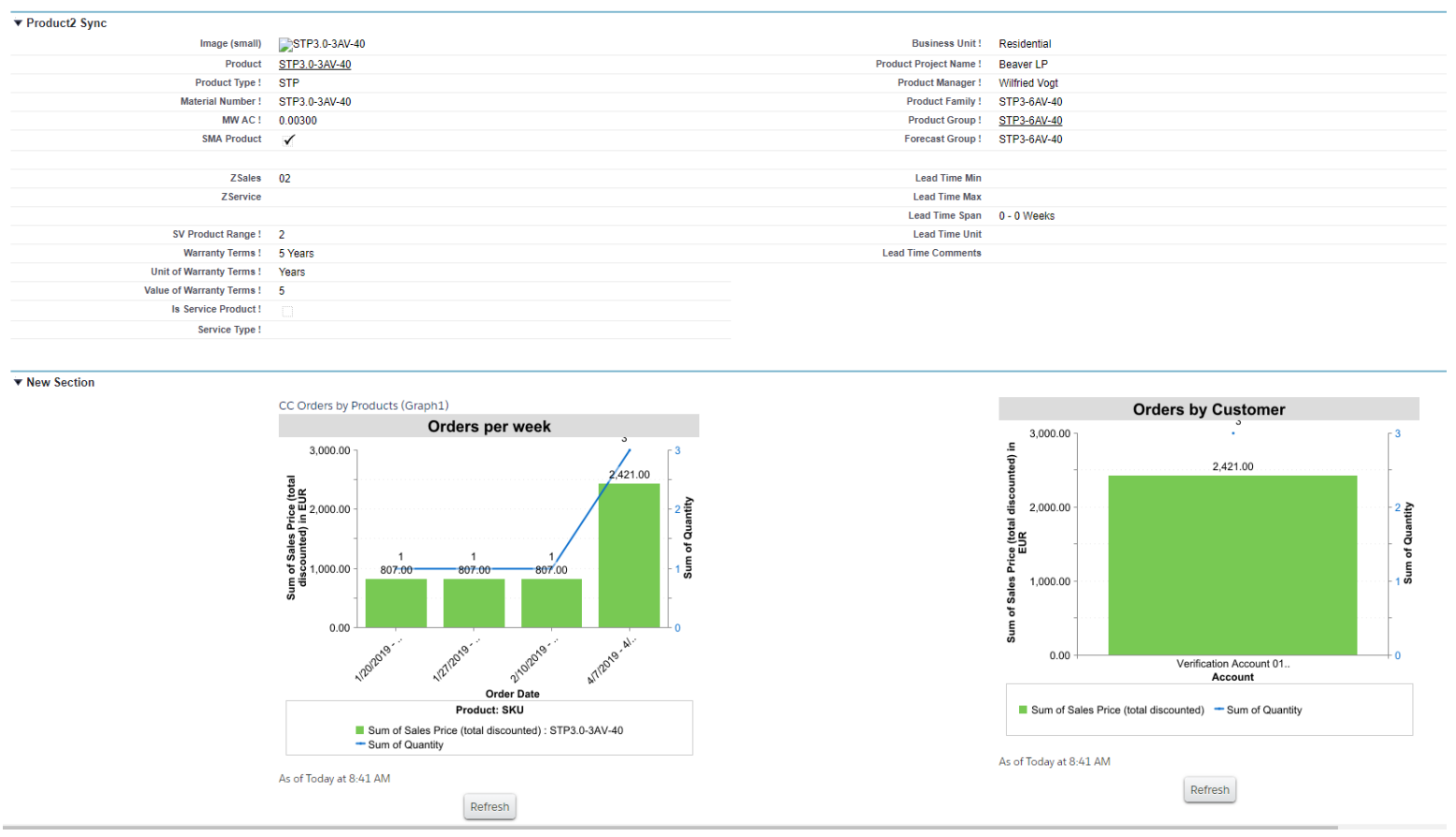

Рис. 13. Графики покупок данного продукта 
Также администратор может видеть всю информацию о заказах пользователя рисунок 14.

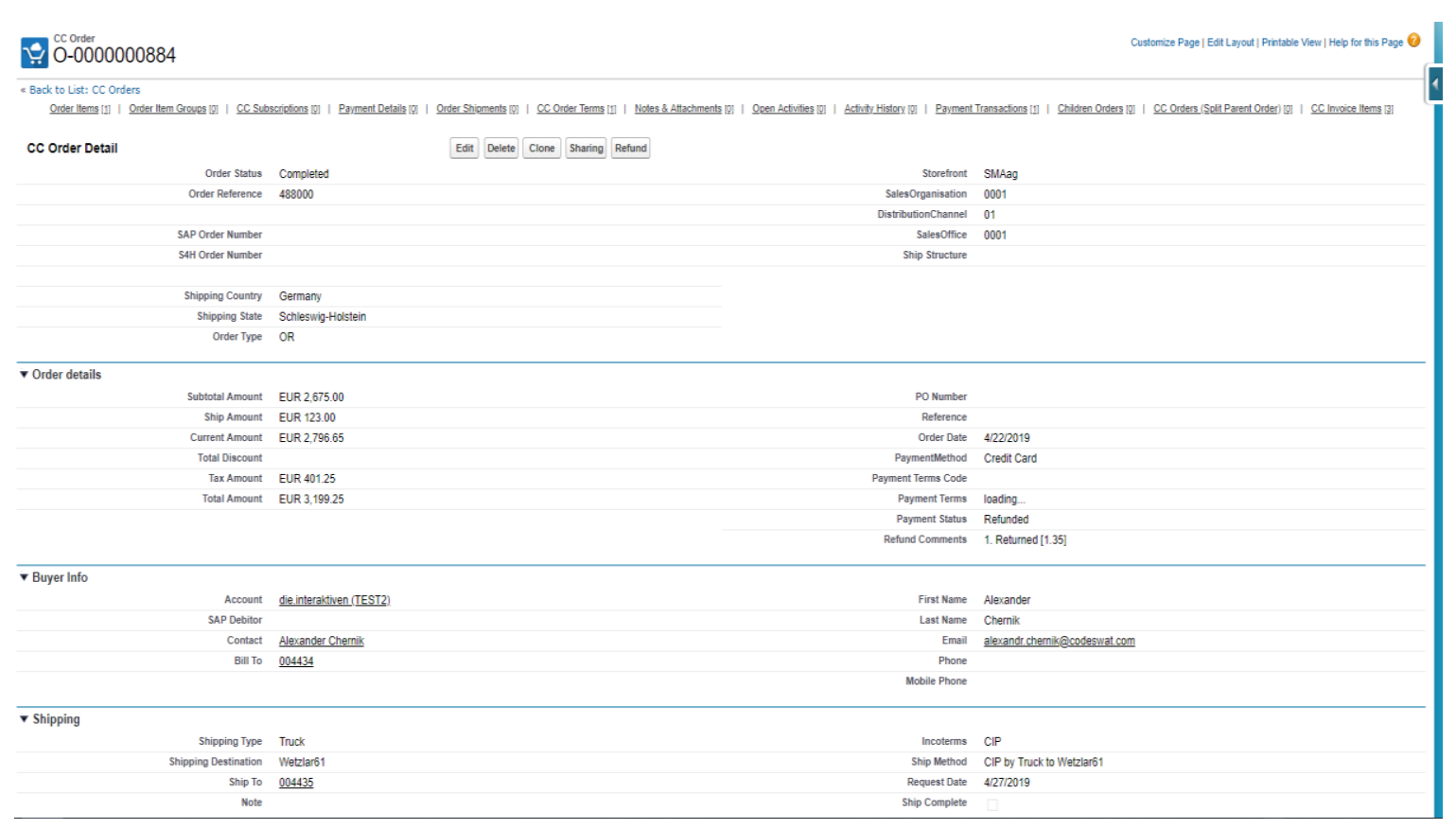

\section{Рис.14. Информация о заказе пользователя}

Ниже указана сумма, которую заплатил пользователь за данный заказ рисунок 15.

\begin{tabular}{|c|c|c|c|c|c|c|}
\hline Notes \& Attachments & New Note Attach file & & & & & Noles \& Allachments Help? \\
\hline \multicolumn{7}{|l|}{ No records to display } \\
\hline SOpen Activities & New Task New Event & & & & & Open Activites Help? \\
\hline \multicolumn{7}{|l|}{ No records to display } \\
\hline StActivity History & Log a Call Wail Merge Send an Email & & & & & Actuity History Help? \\
\hline \multicolumn{7}{|l|}{ No records to display } \\
\hline (1) Payment Transactions & New CC Payment Transaction & & & & & Payment Transactions Help ? \\
\hline Action Payment Transsection Name & Amount velue & is Success & Message & Pyyment Method & Result Code & \\
\hline Edtit Del PT-0202 & EUR 2798000 & $\checkmark$ & & scheme & [retund-recevered] & \\
\hline
\end{tabular}

\section{Рис.15. Сумма заказа пользователя}

Также администратор может выбрать тему отображения самого магазина, что показано на рисунке 16. Тема отображения прописывается непосредственно программистом и после тестирований может быть добавлена в список тем отображения магазина. 


\section{Список литературы}

1. Развитие интернет-торговли в РБ. [Электронный ресурс]. Электронные данные. - Режим доступа: http://edoc.bseu.kz:8080/bitstream/ edoc/61979/1/Yurchenko_126_128.pdf.

2. Science Forum. [Электронный ресурс]. - Электронные данные. Режим доступа: https:// www.scienceforum.ru.

3. Энциклопедия. [Электронный ресурс]. - Электронные данные. Режим доступа: https://www.uchebnikionline.com.

4. Энциклопедия по экономике. [Электронный ресурс]. - Электронные данные. - Режим доступа: https://www.economy-ru.info/info/147998/.

5. VRP Consulting. [Электронный ресурс]. - Электронные данные. Режим доступа: https://www.vrpconsulting.com/.

6. Энциклопедия. [Электронный ресурс]. - Электронные данные. Режим доступа: http://www.marketing.spb.ru/lib-mm/sales/.

7. Шикин, Е.В. Математические методы и модели в управлении: учеб. пособие / Шикин Е.В., Чхартишвили А.Г. - М., 2020 - 440 с.

() А.К. Джамбаев, Л.Т. Курмангазиева, 2021 